\title{
Effect of Index of Consciousness (Ioc2) Monitoring on Remifentanil Dosage During Desflurane Anesthesia: A Randomized Trial
}

\author{
Corresponding Author: \\ Dr. Nirajan Mahaseth \\ Email: drnirajanmht@gmail.com
}

Nirajan Mahaseth ${ }^{1}$, Rabin Khapung ${ }^{1}$, Budal BS ${ }^{2}$

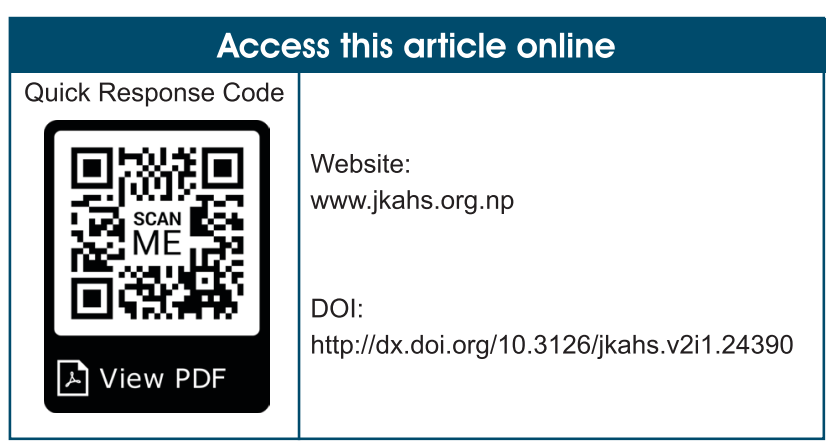

\begin{abstract}
Background: Our study investigated the effects of IOC2 index monitoring on remifentanil dosage during desflurane anesthesia. The objective of this study was to compare the intra-operative events and recovery characteristics between remifentanil adjusted by IOC 2 monitoring guidelines versus remifentanil adjusted by clinical experience according to fluctuation in blood pressure and heart rate during desflurane anesthesia.
\end{abstract}

Methods: Total of 50 consecutive patients of American Society of Anesthesiologists (ASA) status I and II, aged 18-65 years, BMI $18-30 \mathrm{~kg} / \mathrm{m} 2$ undergoing elective laparoscopic cholecystectomy under desflurane anesthesia were enrolled. The patients were randomized and allocated into 2 groups, group $\mathrm{T}$ (Trial group=25) and group $\mathrm{C}$ (control group=25) using sealed envelope method. The infusion rate of remifentanil was adjusted according to IOC2 monitoring guideline between 30 to 50 in Group T whereas the infusion rate of remifentanil is adjusted by clinical experience according to fluctuation in blood pressure in group C. Remifentanil dose, adjustment frequency, infusion duration, intraoperative events, and quality of anesthetic recovery were compared between the two groups.

Results: Mean dose as well as the adjustment frequency of remifentanil was significantly higher in group $\mathrm{T}$ in compared with group $\mathrm{C}(0.1376 \pm 0.0268 \mathrm{vs} 0.0956 \pm 0.0223 \mathrm{mcg} / \mathrm{kg} / \mathrm{min}$ and $5.19 \pm 1.97 \mathrm{vs} 1.7 \pm 0.57 \mathrm{times} /$ surgery, $\mathrm{P}<0.05)$. IOC2 value at the time of skin incision, at the time of gall bladder resection and at the time of skin suture was significantly different between two groups (all $\mathrm{P}$ value $<0.05$ ). Although apparent differences in frequencies of hypotension, bradycardia, hypertension, the total number of patents with intraoperative events were not statistically different between two groups (all $\mathrm{P}>0.05$ ). Both groups had similar voluntary eye opening time, extubation time, emergence agitation, residual sedation 1 and 10 minutes after extubation, post-operative vomiting and NRS score (in PACU and 24 hours after extubation (all $\mathrm{P}>0.05$ ). 
Conclusion: IOC2 data was significantly high when opioid adjusted by clinical experience. The dose of opioid used according to IOC2 (30-50) monitoring guideline utilized higher dose in compared with dose used by clinical experience however, the intraoperative hemodynamic and recovery characteristics was unchanged. Hence, application of IOC2 monitoring might be beneficial to adjust the analgesic depth.

Keywords: IOC2 monitoring, Remifentanil, Desflurane, Laparoscopic cholecystectomy

\section{INTRODUCTION}

Depth of hypnotic component of anesthesia monitors have now been commercially available and are used routinely which enable anesthesiologists to adjust hypnotic agent administration to reduce probability of intraoperative awareness ${ }^{1}$ or discomfort as well as hypnotic overdose which is likely to be involved in postoperative delirium or cognitive dysfunction. EEG (electroencephalographic) monitors such as the bispectral index (BIS) or the state and response entropy index can calculate indexes related to the patient consciousness. Both the technologies have proven their ability to evaluate consciousness of the patient under intravenous (propofol) or volatile agents (sevoflurane) anesthesia.

Compared with other components of anesthesia, no gold standard exists for the assessment of nociception / anti-nociception (NAN) balance, despite several existing technical solutions ${ }^{2}$. Inadequate antinociception often results in blood pressure elevation or tachycardia. The converse, opioid overdose often results in bradycardia and low blood pressure episodes, and has been related to postoperative hyperalgesia ${ }^{2}$. Hence, the search for the optimal opioid administration is a difficult but important objective of modern anesthesia. Analgesic depth is commonly assessed in clinical practice by the patient's clinical signs and symptoms such as blood pressure, heart rate variability, and body movement, but these measures are difficult to renovate into a quantitative standard measure. In addition, some technologies are available for objective monitoring of intraoperative pain, but they suffer from limitations and disadvantages ${ }^{3}$ and body movements can be used as a surrogate for pain ${ }^{4}$. In recent years, the index of consciousness (IOC) has emerged as a new technique for monitoring depth of anesthesia, which not only objectively measures the patient's awareness level ${ }^{5}$ but also reflects analgesic status $^{4}$. Index of consciousness (IOC) consists of IOC1 (index of sedation status) and IOC2 (index of analgesic status).

We hypothesized that IOC2 monitoring would help determine the optimal analgesic status. A recent study investigated the effects of indexes of consciousness (IOC1 and IOC2) monitoring on remifentanil dosage in combination with intravenous agent i.e. propofol however, its effectiveness in combination with inhaled anesthesia was imprecise ${ }^{6}$. In this study, we compared the intraoperative events and recovery characteristics between remifentanil adjusted by IOC2 monitoring guidelines versus remifentanil adjusted by clinical experience according to fluctuation in blood pressure and heart rate during desflurane anesthesia.

\section{METHODS}

Ethical approval was taken from Instutional review committee and the participants involve in the study. Total 70 participants with the age from 18-65 years, BMI $18-30 \mathrm{~kg} / \mathrm{m}^{2}$ undergoing laparoscopic cholecystectomy were assessed for eligibility from September 2016 to January 2017. 20 patients were excluded (17 patient didn't meet inclusion criteria and 3 surgery were cancelled). 50 patients were randomized with sealed envelope, 25 each groups, group $\mathrm{T}$ (Trial group=25) and group $\mathrm{C}$ (control group=25). 2 patient of each group was excluded (1 in each group due to change in surgical method, 1 in each group discontinues intervention). Two patients were lost follow up in trial group whereas three patients lost follow up in control group. Thus, 21 
patients in trial group and 20 patients in control group completed the study and included in the final analysis for group $\mathrm{T}$ and group $\mathrm{C}$ respectively. Participants with pregnancy, allergy to the agents used in the study and with cardio-pulmonary disease were excluded. The change in surgical procedure perioperative or if any anesthetic agents was not used according to study protocol and conditions affecting this trial.

No sedative premedication was administered before surgery. All the participants were routinely monitored with invasive blood pressure, electrocardiogram (ECG), peripheral oxygen saturation, and ETCO2. The group $\mathrm{T}$ received IOC2 monitoring for depth of analgesia (Angel-6000D Multi parameter Anesthesia Monitor, Shenzhen Weihaokang Medical Technology Co., Ltd, Guangdong, and China). The index of depth of analgesic, IOC2, ranges from 0 to 99 and is controlled to be within 30-50 during the operative period, with IOC2 $>50$ indicating insufficient use of analgesic agents and $\mathrm{IoC} 2<30$ indicating excessive analgesic effects.

Both groups were induced with propofol $1.5-2 \mathrm{mg} /$ $\mathrm{kg}$, sufentanil $0.5 \mathrm{mcg} / \mathrm{kg}$ and vecuronium $0.1 \mathrm{mg} / \mathrm{kg}$ and tracheal intubation was performed for mechanical ventilation when satisfactory muscle relaxation was achieved (about 3 minutes), followed by connection to an anesthetic machine (Dräger fabius GS) for volume controlled ventilation with a tidal volume of $8 \mathrm{ml} / \mathrm{kg}$, a respiratory rate of $12 / \mathrm{min}$, a respiratory ratio of $1: 2$, and a pressure of end-tidal carbon dioxide (PETCO2) of 35-45 mmHg. Anesthesia was maintained with desflurane end tidal concentration 0.8-1.1 MAC and remifentanil. For muscle relaxation, Vecuronium $0.015 \mathrm{mg} / \mathrm{kg}$ was repeated after 45 minutes. Remifentanil was prepared in $20 \mathrm{ml}$ syringe and diluted to $50 \mathrm{mcg} / \mathrm{ml}$ and continuously infused over maintenance period via syringe pump. The infusion rate of remifentanil was adjusted according to IOC2 monitoring guideline in Group T $(n=25)$ whereas the infusion rate of remifentanil is adjusted by clinical experience according to fluctuation in blood pressure and heart rate in group $\mathrm{C}(\mathrm{n}=25)$. In group $\mathrm{T}$, the infusion rate of remifentanil was increased by $2 \mathrm{ml}$ $(100 \mathrm{mcg})$ per adjustment when IOC2 $>50$ and was decreased by $2(100 \mathrm{mcg})$ per adjustment when IOC2 $<30$, with the maintenance value between 30 and 50. The dose of remifentanil was adjusted by clinical experience according to fluctuation in blood pressure and heart rate so as to control the fluctuation of blood pressure and heart rate within $20 \%$ of baseline values. For each adjustment of remifentanil, infusion rate was increased or decreased by $2 \mathrm{ml}(100 \mathrm{mcg})$. Tropisetron $2 \mathrm{mg}$ and pericoxib sodium $40 \mathrm{mg}$ was administered 5 minutes before the end of surgery for prevention of nausea and vomiting and postoperative pain respectively. To minimize the risk of residual neuromuscular blockage risk after the operation, decurarization has been provided by neostigmine $0.01 \mathrm{mg} / \mathrm{kg}$ and atropine $0.01 \mathrm{mg} / \mathrm{kg}$. The following indexes were recorded:

1. General patient data (age, sex, weight, height, BMI),

2. Remifentanil (adjustment frequency of target concentration, infusion duration, and mean dosage,

3. Blood pressure, and heart rate (baseline and intraoperatively at different surgical events,

4. IOC2 values at different surgical events,

5. Frequencies of intraoperative events (hypertension, hypotension, tachycardia, bradycardia, and number of patients with intraoperative events) and

6. Quality of anesthetic recovery (voluntary eye opening time, extubation time, Richmond agitation and sedation score, post-operative vomiting and NRS score for post-operative pain)

All data were analyzed using SPSS 24.0. Continuous data are shown as mean \pm standard deviation (SD). Between-group comparisons were performed using the independent sample $t$ test or the rank sum test. Categorical data were compared using the chi-square test. A P value $<0.05$ was considered statistically significant. 


\section{RESULTS}

Table 1: Comparison of general data between the two groups (mean \pm SD)

\begin{tabular}{lccc}
\hline & Group T & Group C & P value \\
\hline Age (years) & $48.57 \pm 11.76$ & $39.95 \pm 13.12$ & 0.032 \\
Sex (F/M)* & $12 / 9$ & $11 / 9$ & $0.570^{*}$ \\
Weight (kilogram) & $62.05 \pm 6.79$ & $62.20 \pm 9.38$ & 0.953 \\
Height (centimeter) & $161.81 \pm 7.76$ & $161.25 \pm 8.81$ & 0.830 \\
BMI (kg/m $\left.{ }^{2}\right)$ & $23.79 \pm 2.98$ & $23.88 \pm 2.71$ & 0.916 \\
\hline
\end{tabular}

*compared using chi square test, BMI: body mass index

As shown in table 1, two groups were not significantly different in age, height, weight, BMI. We choose the adult patients between 18 to 65 years old in both groups so there was no any significant difference with age.

\section{Table 2: Comparison of use of remifentanil between the two groups (mean \pm SD)}

\begin{tabular}{lccc}
\hline \multicolumn{1}{c}{ Items } & Group T & Group C & P value \\
\hline Mean dose of Remifentanil (mcg/kg/min) & $0.1376 \pm 0.0268$ & $0.0956 \pm 0.0223$ & 0.000 \\
Infusion duration of remifentanil (Minutes) & $79.57 \pm 34.05$ & $75.65 \pm 24.47$ & 0.676 \\
Dose adjustment frequency of Remifentanil (times/surgery) & $5.19 \pm 1.97$ & $1.7 \pm 0.57$ & 0.000 \\
\hline
\end{tabular}

Values are the number of patients (\%), mean (standard deviation), or the median (interquartile range).

As shown in table 2, remifentanil infusion duration was not significantly different between the two groups ( $\mathrm{P}>$ $0.05)$, but and mean dosage and the adjustment frequency of remifentanil were significantly different between 2 groups $(\mathrm{P}<0.05)$.

Table 3: Comparison of IOC2 value between the two groups (mean \pm SD)

\begin{tabular}{lccc}
\hline \multicolumn{1}{c}{ IOC2 value } & Group T & Group C & P value \\
\hline At the time of skin incision & $36.29 \pm 10.29$ & $49.95 \pm 12.91$ & 0.001 \\
At the time of GB resection & $42.10 \pm 9.69$ & $53.75 \pm 11.48$ & 0.001 \\
At the time of skin suture & $40.10 \pm 8.26$ & $52.50 \pm 10.56$ & 0.000 \\
\hline
\end{tabular}

As shown in table 4, IOC2 value at the time of skin incision, gall bladder resection and skin suture was significantly different between two groups (all $\mathrm{P}$ value $<0.05$ ). 
Table 4: Intra-operative events in the two groups

\begin{tabular}{lccc}
\hline \multicolumn{1}{c}{ Item } & Group T (n=21) & Group C (n=20) & P value \\
\hline Hypotension & $8(38.09 \%)$ & $9(45 \%)$ & 0.448 \\
Bradycardia & $8(38.09 \%)$ & $9(45 \%)$ & 0.448 \\
Hypertension & $4(19.04 \%)$ & $3(15 \%)$ & 0.529 \\
Tachycardia & $1(4.7 \%)$ & $2(10 \%)$ & 0.481 \\
Number of patients & & & \\
with intraoperative events & $11(52.38 \%)$ & $13(65 \%)$ & 0.308 \\
Intraoperative awareness & 0 & 0 & 1.00 \\
Events per patient & 1.57 & 1.90 & \\
\hline
\end{tabular}

As shown in table 4 (intra-operative events), although some apparent differences in frequencies of hypotension (38.09\%vs $45 \%, \mathrm{P}=0.448)$, bradycardia ( $38.09 \%$ vs $45 \%, \mathrm{P}=0.448)$, hypertension ( $19.04 \%$ vs $15 \%, \mathrm{P}=0.529$ ), tachycardia ( $4.7 \%$ vs $10 \%, \mathrm{P}=0.481)$, and number of patients with events $(52.38 \%$ vs $65 \%, \mathrm{P}=0.308)$ could be observed, they were not statistically significant (all $\mathrm{P}>0.05$ ) intraoperative events per patient were 1.57 and 1.90 in group $\mathrm{T}$ and group $\mathrm{C}$ respectively.

Table 5: Comparison of quality of anesthetic recovery between the two groups

\begin{tabular}{lccc}
\hline \multicolumn{1}{c}{ Items } & Group T & Group C & P value \\
\hline Voluntary eye opening (minutes) & $10.48 \pm 1.68$ & $9.80 \pm 1.72$ & 0.210 \\
Extubation time (minutes) & $14.00 \pm 2.02$ & $13.35 \pm 2.08$ & 0.317 \\
Emergence agitation (no. of patients) & $2(9.5 \%)$ & $3(15 \%)$ & 0.476 \\
Residual sedation (1 minutes after extubation) & $4(19.04 \%)$ & $2(10 \%)$ & 0.355 \\
Residual sedation (10 minutes after extubation & 0 & 0 & 1.00 \\
Early Post-operative vomiting (0-4 hours) & 0 & $2(10 \%)$ & 0.232 \\
Late post-operative vomiting (4-24 hours) & $4(19.04 \%)$ & $8(40 \%)$ & 0.129 \\
NRS score (assessment at PACU) (mild/moderate/severe) & $11(52 \%) / 0 / 0$ & $14(70 \%)$ & 0.29 \\
NRS score (assessment at 24 hours after surgery) & $6(28.57 \%) / 0 / 0$ & $3(15 \%) / 0$ & 0.222 \\
(mild /moderate/severe) & & $9(45 \%) / 0 / 0$ & \\
\hline
\end{tabular}

Overall and severe emergence agitation was defined as Richmond agitation-sedation scale (RASS) score $\geq+2$ and $\geq+3$, respectively. Residual sedation was defined as RASS $\leq-2$. NRS: numerical rating scale $(0=$ No pain, $1-3=$ mild pain, $4-6=$ moderate pain, $7-10=$ severe pain)

As shown in table 5, Both groups had similar voluntary eye opening, extubation time, emergence agitation, residual sedation 1 and 10 minutes after extubation, post-operative vomiting and NRS score (in PACU and 24 hours after extubation (all $\mathrm{P}>0.05$ ). 


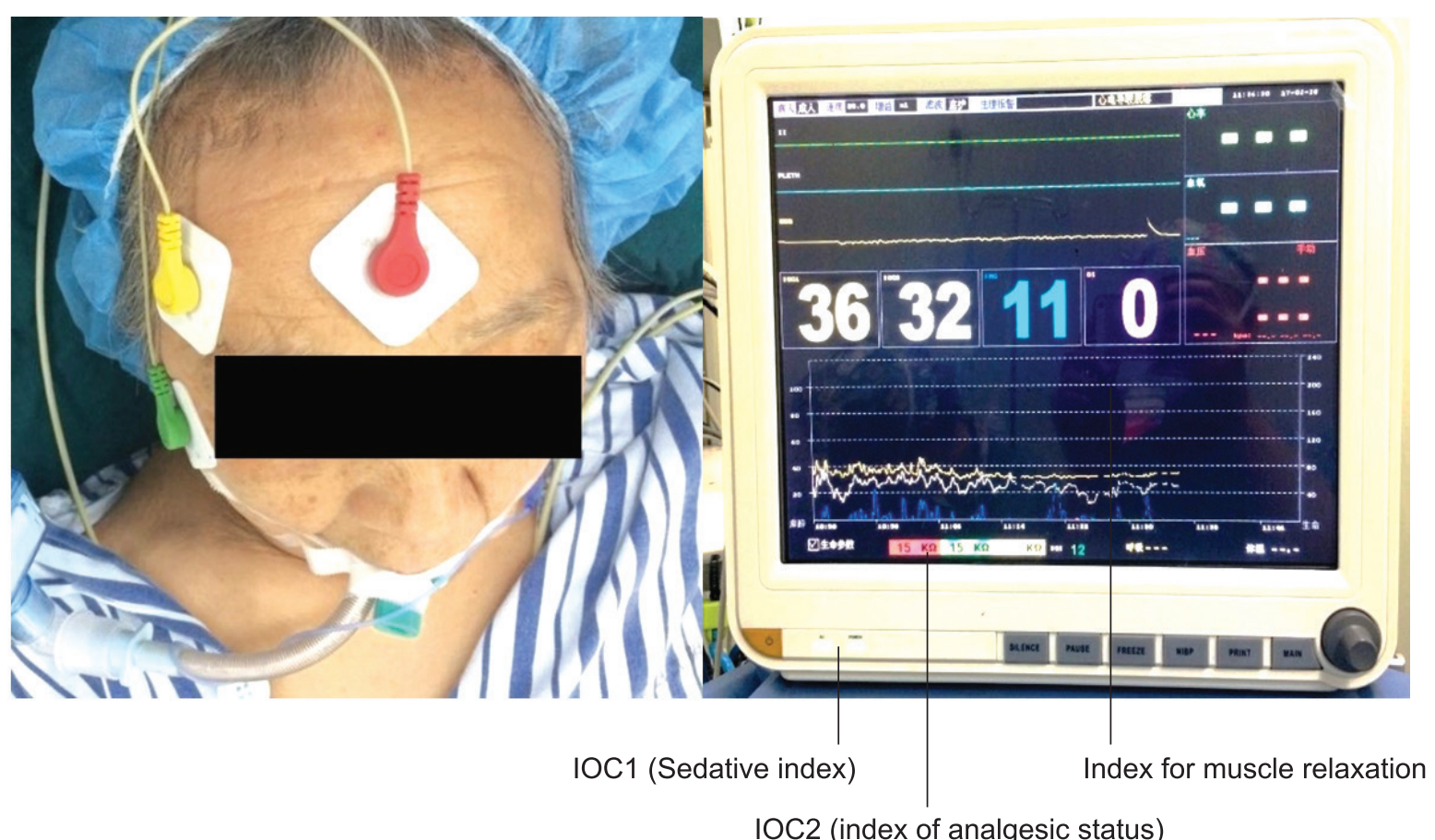

Figure 1: IOC2 index monitor (Angel-6000D Multiparameter Anesthesia Monitor, Shenzhen Weihaokang Medical Technology Co., Ltd, Guangdong, and China)

\section{DISCUSSION}

With the widespread use of target-controlled infusion and anesthesia depth monitoring, accurate anesthesia is gradually becoming possible ${ }^{7,8}$. We studied the effects of IOC2 monitoring on remifentanil dosage compared with remifentanil used by clinical experience according to fluctuation in blood pressure and heart rate during desflurane anesthesia. Our results showed that the mean dose of remifentanil and syringe pump adjustment frequency of remifentanil was significantly higher in group $\mathrm{T}$ in compared with group $\mathrm{C}$. There was no significant difference in infusion duration of remifentanil between two groups. The baseline blood pressure and heart rate was not significantly different between two groups. Similarly the intraoperative systolic blood pressure (SBP), diastolic blood pressure (DBP) heart rate (HR) at different surgical events time was not significantly different between two groups. However, in compared with group T, IOC2 value was significantly higher in group $\mathrm{C}$ at the time of different surgical events which gives the suspicion that the dose of opioid we use according to clinical experience might be insufficient. A similar study by Guisheng Wu et al. ${ }^{6}$ also concluded that the use of IOC2 might be applied to guide the dose of remifentanil. Voluntary eye opening, extubation time, emergence agitation, residual sedation, post-operative vomiting and pain score were not significantly different between the two groups. however there was slight reduction in the frequency of emergence agitation, post-operative vomiting and NRS score for pain but still statically not significant. The adverse events such as hypotension, bradycardia, hypertension, tachycardia, body movement and number of patients with adverse events were also not significantly different between two groups. Although study by Guisheng Wu et al. ${ }^{6}$ showed the reduction of occurrence of adverse events and better recovery however our study showed no significant difference between 2 groups.

The doses of remifentanil were adjusted based on the patient's baseline blood pressure and heart rate in group $\mathrm{C}$ to make them swing within $80-120 \%$ of baseline values during surgery. Remifentanil used according to IOC2 monitoring guideline (3050), mean dose of remifentanil and the adjustment frequency were higher in the group $\mathrm{T}$ than in the group $\mathrm{C}$, but both group showed similar frequencies 
of hypertension, hypotension. This revels the remifentanil used according to IOC2 the guideline of monitor utilizes high dose of remifentanil however, such high dose do not effect much in hemodynamic stability, intraoperative events and post-operative recovery.

Similar studies done by Huber and Net etal have revealed that pain can induce changes in $\mathrm{EEG}$ power, ${ }^{9,10}$. Jensen et al. ${ }^{4}$ confirmed that IoC1 (qCON) can reliably predict the disappearance of eyelash reflex (or, the disappearance of awareness) during TIVA using propofol and remifentanil. Moreover, with similar concentrations of anesthetics, IoC2 (qNOX) can predict the occurrence of body movements to nociceptive stimuli. In the present study, nociceptive stimuli mainly occurred during anesthesia or surgical procedures such as tracheal intubation/extubation, skin incision, and gall bladder resection.

Nociception stimuli perceived during surgical procedure under the influence of some level of analgesia do not influence much on hemodynamic but our monitor which receive EEG signals to convert IOC2 index expresses the inadequacy of analgesic component of anesthesia more sensitively and quickly. Similar to other EEG curve, IoC2 curve is not smooth during the maintenance of anesthesia. This reveals that analgesic depth of anesthesia is essentially a state of the central nervous system that is affected by the interactions between the irritations from nociceptive stimuli and the inhibitory effects of anesthetic agents. In other words, it is a functional state of the central nervous system occurring when surgical stimulation dynamically balances against the control effects of general anesthetics, indicating that IoC2 will still slightly fluctuate due to surgical stimuli although depth of anesthesia is relatively stable. According to our clinical experience, the doses of remifentanil should be changed when IOC2 increases or decreases lower than their reference range for 2 minutes to avoid frequent adjustment.

This study suffers from some limitations. The sample size was small and surgery is too short. Sufentanil was used for intubation, but the same dose was used in both groups, therefore, it should not influence the comparison between the two groups. Importantly, desflurane administration was not standardized and was based on MAC, therefore introducing variability that could mask the real effects of IOC2 monitoring. Finally, some confounding factors that may affect our results have not been controlled so, additional studies are necessary to examine.

\section{CONCLUSION}

IOC2 data was significantly high when opioid adjusted by clinical experience. The dose of opioid used according to IOC2 (30-50) monitoring guideline utilized higher dose in compared with dose used by clinical experience however, the intraoperative hemodynamics and recovery characteristics was unchanged. Hence, application of IOC2 monitoring might be beneficial to adjust the analgesic depth.

\section{REFERENCES}

1. Myles PS, Leslie K, Mcneil JJ, Forbes A, Chan MT. Bispectral index monitoring to prevent awareness during anaesthesia: the B-Aware randomised controlled trial. Acc Current Journal Review 2004; 13(8):35-39.

2. Joly V, Richebe P, Guignard B, Fletcher D, Maurette P, Sessler DI, Chauvin M. Remifentanil-induced postoperative hyperalgesia and its prevention with small-dose ketamine. Anesthesiology 2005;103(1):147155 .

3. De Jonckheere J, Bonhomme V, Jeanne M, Boselli E, Gruenewald M, Logier R,et al. Physiological signal processing for individualized anti-nociceptionmanagement during general anesthesia: a review. Yearb Med Inform 2015;10:95-101.

4. Jensen EW, Valencia JF, Lopez A, Anglada T, Agusti M, Ramos $\mathrm{Y}$ et al. Monitoring hypnotic effect and nociception with two EEGderived indices, qCON and qNOX, during general anaesthesia. Acta Anaesthesiologica Scandinavica 2014;58(8), 933-941. 
5. Kempen PM. Let us make MRI anesthesia safe! Anesth Analg. 2007; 104:1602-9.

6. Wu G, Zhang L, Wang X, Yu A, Zhang Z, Yu J. Effects of indexes of consciousness ( $\mathrm{IoC} 1$ and $\mathrm{IoC} 2$ ) monitoring on remifentanil dosage in modified radical mastectomy: a randomized trial. Trials2016;17(1):25-29.

7. Shoushtarian M, Sahinovic MM, Absalom AR, Kalmar AF, Vereecke HE, Liley DT, et al. Comparisons of electroencephalographically derived measures of hypnosis and Antinociception in response to standardized stimuli during target-controlled Propofolremifentanil anesthesia. Anesth Analg. 2016; 122(2):382-92.

8. Shalbaf R, Behnam H, Sleigh JW, Steyn-Ross A, Voss LJ. Monitoring the depth of anesthesia using entropy features and an artificial neural network. J Neurosci Methods. 2013;218(1):1724.
9. Nir RR, Sinai A, Moont R, Harari E, Yarnitsky D. Tonic pain and continuous EEG: prediction of subjective pain perception by alpha-1 power during stimulation and at rest. Clin Neurophysiol. 2012;123:605-12.

10. Huber MT, Bartling J, Pachur D, WoikowskyBiedau S, Lautenbacher S. EEG responses to tonic heat pain. Exp Brain Res. 2006;173:1424.

\section{How to cite this article?}

Mahaseth N, Khapung R, Budal BS. Effect of Index of Consciousness (Ioc2) Monitoring on Remifentanil Dosage During Desflurane Anesthesia: A Randomized Trial. Journal of Karnali Academy of Health Sciences. 2019; 2 (1): 10-17

Source of Support: Self, Conflict of Interest: None declared 